\title{
Functional Improvement after the Gross Motor Function Measure-88 (GMFM-88) Item-Based Training in Children with Cerebral Palsy
}

\author{
Jooyeon Ko \\ Department of Physical Therapy, Daegu Health College, Daegu, Korea
}

\begin{abstract}
Purpose: The aim of this study was to investigate applicability of the GMFM-88 in planning intervention for CP children. Specifically, this study assessed functional improvement after a four-week GMFM-88 item-based training in CP children divided into three age groups ( $\leq 24$ months, 25-48 months, and $>48$ months) and five levels of the gross motor function classification system (GMFCS).

Methods: Subjects were 264 children with CP (mean age 32.90 months) recruited from one CP clinic. The GMFM-88 item-based training was planned for each child, after an interview with its caregiver. To investigate functional improvement after the intervention, minimum important difference (MID) and MID proportion for the change in scores of GMFM-88 were calculated.

Results: The GMFM-88 scores increased after the interventions in all three age groups $(p<0.05)$. In particular, children with CP aged $\leq 24$ months and at the GMFCS level II showed greater functional improvement after training.

Conclusion: This study found that the GMFM-88 item-based training would be used to plan activity-oriented intervention both in clinic and home in each CP child.
\end{abstract}

Keywords: Cerebral palsy, GMFM-88, Minimum important difference

\section{INTRODUCTION}

Cerebral palsy (CP) is a group of movement and posture disorders, causing activity limitation in childhood. ${ }^{1}$ The primary aim of rehabilitation therapy for children with CP is to improve motor skills necessary for daily life activities. Evidence indicates that interventions including task-oriented training are the best approach for treatment of children with $\mathrm{CP}^{2}$ Task-oriented training for $\mathrm{CP}$ involves an individualized activity-based approach. ${ }^{3}$ This, however, requires defining a specific goal, describing outcomes for this goal on a scale, selecting a measure for detecting clinically important changes, and evaluating the subject's change in gross motor function following intervention. ${ }^{3}$

The gross motor function measure-88 (GMFM-88) scale is used to plan treatment and detect quantitative changes in gross motor function in children with $\mathrm{CP}^{4,5}$ In a study describing daily physical activity levels, the GMFM-E (walking, running, and jumping) dimension significantly correlated with daily physical activity. ${ }^{6}$ The authors of this earlier study suggested that task-specific practice on the GMFM-E dimension may lead to improved daily activity in children with $\mathrm{CP}$ at gross motor function classification system (GMFCS) levels II and III. In another study on the effectiveness of functional task-oriented programs for children with mild to moderate $\mathrm{CP}$, researchers set a maximum of three different activities for each long-term goal. One activity was to walk in and around the house without falling. The long-term goal was divided into three relevant, but less complex, short-term goals such as stepping over a stick with the right/left foot or kicking a ball with the right/left foot, which were all GMFM-E dimension items?

A single-blinded, randomized controlled trial reported by Salem and Godwin ${ }^{8}$ subsequently examined the effects of practicing functional task training with 10 children with CP, at GMFCS levels I-III, 
twice a week for five weeks. The task-specific training was composed of activities similar to those the child normally performs as part of a daily routine in the home and community such as walking forward, backward, and sideways, sit-to-stand transitions, stair climbing, kicking a ball, and single leg stance. All of the tasks provided to the children in this study were items of dimension $\mathrm{D}$ (standing) and E (walking, running and jumping) of the GMFM-88 scale, and the authors also used these two dimensions as primary outcome measure in their study.

The minimum important difference (MID) and MID proportion are used to detect functionally meaningful changes after interventions at a group or individual levels, respectively. ${ }^{9-11}$

This study investigated how the GMFM- 88 scale can be used for planning intervention strategies and detecting functional improvement in motor activities of children with CP. Specifically, the purpose of this study was to investigate the MID of the GMFM-88-goal total and total scores after 4-weeksof GMFM-88 items-based training in children with CP.

\section{METHODS}

\section{Subjects}

In total, 264 children with CP (mean age 32.9 \pm 21.6 months, 137 boys, 127 girls) were recruited between 2013 and 2014 from a CP clinic in South Korea. The participating researcher and research assistants obeyed the Helsinki declaration during the study. All caregivers were informed of the procedures and the purposes of this study, and all signed informed consent forms. The inclusion criteria were as follows: confirmed diagnosis of CP through clinical examinations; absence of nerve block injections, such as a Botox within the previous 4 months and an absence of orthopedic surgery, such as muscle-lengthening surgery within the previous 6 months.

\section{Experimental methods}

\section{1) Measurement}

(1) Gross motor function classification measure (GMFCS)

The subjects were classified by GMFCS into five levels (I: walks without limitations, II: walks with limitations, III: walks using a hand-held mobility device like walker, crutch, cane, IV: self-mobility with limitations and may use powered wheelchair, and V: transported in a manual wheelchair by others) according to motor func- tion. In this study, the Korean version of the GMFCS was used.

\section{(2) Gross motor function measure-88 (GMFM-88)}

The gross motor function measure-88 (GMFM-88) is a well-known scoring system, which assesses gross motor development in children with CP over time. The GMFM does not have age limits, and consists of 88 items categorized into five gross motor function dimensions: A (lying and rolling), 17 items; B (sitting), 20 items; C (crawling and kneeling), 14 items; D (standing), 13items; and E (walking, running, and jumping), 24 items. Each item is scored on a fourpoint scale (0-dose not initiate the task, 1-initiates the task, 2-partially completes the task, 3-completes the task). The raw score for each dimension was converted into a percentage score, and each dimension was equally weighted. The most frequently used parameters in the GMFM-88 are the GMFM-88-goal total and total scores. ${ }^{10,12}$

In the present study, the GMFM-88-goal total score as well as total score were used. The goal total score was calculated as the mean of the dimension scores selected, and the total score was calculated by summing the percentages of the five dimensions and dividing the result by five, the scores ranged from 0 to 100. A higher score represents better gross motor.4 In the present study, the Korean version of the GMFM-88 (K-GMFM-88) was used. The inter-rater reliability of the K-GMFM-88 was assessed by interclass correlation coefficient (ICC), ${ }^{13-16}$ which ranged from 0.975-0.997. Test-retest reliability over a two-week intervalhas been shown to be in the range of 0.998 to $1.000 .^{10}$

\section{2) Procedures}

Gross motor function and the GMFCS levels of CP were assessed with the help of each child's therapist. The total number of the pediatric therapists was four and each of whom had more than three years clinical experience in administering the GMFM-88 and the GMFCS.

All participants were scheduled to participate in two separate test sessions using the K-GMFM-88 scale before and after the onemonth period of intensive training to examine group and individual levels of meaningful gross motor changes.

\section{3) Interventions}

Each child's therapist established treatment strategies using the GMFM-88 items in collaboration with the child's caregiver. For ex- 
ample, for children who were $\leq 24$ months old at GMFCS levels I, II, and III, all five dimensions of the GMFM- 88 were of interest to the therapists and caregivers, while for children at levels IV and V, only dimension A or B were of interest. Pediatric therapists and caregivers tried to link GMFM-88 items to each child's daily life. For example, item 54 or 55 (standing: holding on to a large bench with one hand, lifts right or left foot for 3 seconds) in dimension D was selected frequently because the items were related to wearing shoes, holding furniture, or crossing over obstacles in real life. During the intervention period, each child was given therapy over two sessions (30 minutes per session) per day, five days a week for four weeks.

\section{4) Statistical analysis}

Analyses were performed using SPSS (Version 12.0.1; SPSS Inc., Chicago, IL, USA). After examining the normal distribution of the data with a Kolmogorov-Smirnov test, means and standard deviations (SD) of the GMFM-88 scores were calculated. The paired t-test was used to measure the statistical significance of the change in scores over the one-month period. To determine functionally meaningful change, the minimum important difference (MID) and the MID proportion (\%) were calculated. The MID is a statistical index to represent group level of meaningful change, while MID proportion is on an individual level after interventions. The MID is the lower boundary of change that has been defined as clinically important. ${ }^{17}$ Any amount of change greater than the MID threshold is considered meaningful. The MID was calculated by using three commonly used effect size of $0.3,0.5$, and 0.8 which represents small, moderate, and large improvement respectively: MID
$(0.3)=0.3 \times \mathrm{SD}_{\mathrm{b}}, \mathrm{MID}(0.5)=0.5 \times \mathrm{SD}_{\mathrm{b}}$, and $\mathrm{MID}(0.8)=0.8 \times \mathrm{SD}_{\mathrm{b}}$, where $\mathrm{SD}_{\mathrm{b}}$ is the standard deviation of the baseline scores. ${ }^{18}$ The three MID proportions are also defined as the percentage (\%) of participants who exceed the MID (0.3), MID (0.5), and MID (0.8) values. $^{19}$

\section{RESULTS}

The general characteristics of the participants are shown in Table 1. The results of the interviews for matching the GMFM-88 items to each child's activity are shown in Table 2.

The results of the paired t-test for the data obtained before and after one month of intervention showed significant differences in all three age groups and across all five GMFCS levels $(\mathrm{p}<0.05)$ (Tables 3 and 4), except for children $>48$ months at GMFCS levels II and III.

To interpret MIDs of the GMFM-88-goal total and total scores, mean change values before and after intervention were used as a reference score. For example, the MIDs of the GMFM-88- goal total in children $\leq 24$ months at GMFCS level I showed a mean change of 9.44point. MID (0.3), MID (0.5), and MID (0.8) were 4.52, 7.53, and 12.05, respectively (Table 3). The MID proportions of the GMFM-88-goal total score in children $\leq 24$ months at GMFCS level I were as follows: $75 \%, 62.5 \%$, and $54.17 \%$ (18, 15, and 13 children) (Table 3). The MIDs of the GMFM-88-total score in children $\leq 24$ months at GMFCS level I showed a mean change of 6.93 point. MID (0.3), MID (0.5), and MID (0.8) were 5.96, 9.93, and 15.88, respectively (Table 3). The MID proportions of the GMFM-88-total score in children $\leq 24$ months at GMFCS level I were as follows:

Table 1. General characteristics of the sample

\begin{tabular}{|c|c|c|c|c|}
\hline Variables & $\leq 24$ months $(n=97)$ & $25-48$ months $(n=114)$ & $>48$ months $(n=53)$ & Total $(n=264)$ \\
\hline \multicolumn{5}{|l|}{ Sex } \\
\hline Boys & $51(52.6)$ & $60(52.6)$ & $26(49.1)$ & $137(51.9)$ \\
\hline Girls & $46(47.4)$ & $54(47.4)$ & $27(50.9)$ & $127(48.1)$ \\
\hline \multicolumn{5}{|l|}{ GMFCS levels } \\
\hline I & 24 & 21 & 10 & $55(20.8)$ \\
\hline$\|$ & 15 & 20 & 10 & $45(17.0)$ \\
\hline III & 14 & 20 & 4 & $38(14.4)$ \\
\hline IV & 19 & 31 & 14 & $64(24.2)$ \\
\hline V & 25 & 22 & 15 & $62(23.5)$ \\
\hline Age (months) & $14.0 \pm 5.6$ & $33.5 \pm 6.9$ & $66.3 \pm 19.8$ & $32.92 \pm 21.6$ \\
\hline
\end{tabular}

Values are presented as $n$ (\%) or mean \pm standard deviation.

GMFCS: gross motor function classification system. 
Table 2. Matching the GMFM-88 items to CP child's daily activities

\begin{tabular}{|c|c|c|}
\hline GMFM-88 dimensions & Items & Examples of matching daily activities \\
\hline A (Lying \& rolling) & $\begin{array}{l}\text { 6/7. Supine: Reach out with } R / L \text { arm, hand crosses midline toward } \\
\text { toy }\end{array}$ & - Flipping over a book page \\
\hline \multirow[t]{6}{*}{ B (Sitting) } & 19/20. Supine: Roll to R/L side, attains sitting & - Sitting up after rotating the body from lying on the bed \\
\hline & $\begin{array}{l}\text { 21. Sit on mat, supported at thorax by therapist: Lift head upright, } \\
\text { maintain } 3 \text { seconds }\end{array}$ & - Basic posture for observing an object, speaking, and eating \\
\hline & 33. Sit on mat: Pivots $90^{\circ}$, without arms assisting & - Grapping a nearby block when the kid plays block games \\
\hline & 34. Sit on bench: maintains, arms and feet free, 10 seconds & - Sitting on a chair for reading \\
\hline & 35. Standing: Attains sit on small bench & - Sitting on a low-height chair at a kindergarten or at home \\
\hline & 37. On the floor: Attains sit on large bench & - Going up to bed \\
\hline \multirow[t]{2}{*}{ C (Crawling \& kneeling) } & $\begin{array}{l}\text { 46/47. } 4 \text { point: Crawls up/backward down } 4 \text { steps on hands and } \\
\text { knees/feet }\end{array}$ & - Up \& down a Jungle Gym or a rock at the playground \\
\hline & 51. High kneeling: kneeling walks forward 10 steps, arms free & - Delivering or tossing an object to another person \\
\hline \multirow[t]{6}{*}{ D (Standing) } & 54/55. Standing: Holding on to large bench with one hand, lifts & - Wearing trousers with gripping mom's shoulder \\
\hline & $R / L$ foot, 3 seconds & - Wearing shoes with standing \\
\hline & 56. Standing: Maintains, arms free, 20 seconds & - (a boy kid) Peeing with standing \\
\hline & 57/58. Standing: Lift L/R foot, arms free, 10 seconds & - One-leg standing when wearing trousers \\
\hline & & - One-leg standing when the kid cross an obstacle or kicking a ball \\
\hline & 63. Standing: Attains squat, arms free & - Squatting down to draw something on the ground \\
\hline \multirow[t]{9}{*}{ E (Walking, running \& jumping) } & 69. Standing: Walks forward 10 steps & - All daily walking activities \\
\hline & $\begin{array}{l}\text { 72. Standing: Walks forward } 10 \text { steps, carrying a large object with } \\
2 \text { hands }\end{array}$ & - Moving with holding a big book or a toy car \\
\hline & $\begin{array}{l}\text { 74. Standing: Walks forward } 10 \text { consecutive steps on a straight } \\
\text { line } 2 \mathrm{~cm} \text { wise }\end{array}$ & t - Walking on the balance beam at the playground or school \\
\hline & 75/76. Standing: Steps over stick at knee level, R/L foot leading & - Crossing or Walking on the sidewalk block \\
\hline & & - Walking into a baby tub by oneself \\
\hline & 78/79. Standing: Kicks ball with R/L Foot & - Playing a ball game with friends \\
\hline & 80. Standing: Jumps $30 \mathrm{~cm}$ high, both feet Simultaneously & - Jumping rope \\
\hline & 86/87. Standing: Walks up/down 4 steps, alternating feet & - Up \& down stairs \\
\hline & 88. Standing on $15 \mathrm{~cm}$ step: Jumps off, both feet simultaneously & - Jumping from a sofa or a stair \\
\hline
\end{tabular}

GMFM: gross motor function measure, R: right, L: left, 4-point: quadraped position.

Table 3.MID and MID proportion of GMFM-88 after intervention for CP aged $\leq 24$ months

\begin{tabular}{|c|c|c|c|c|c|c|c|c|c|}
\hline & $\begin{array}{l}\text { 1st test } \\
M \pm S D\end{array}$ & $\begin{array}{l}\text { 2nd test } \\
M \pm S D\end{array}$ & $\begin{array}{l}\text { Change } \\
M \pm S D\end{array}$ & $\begin{array}{l}\mathrm{MID}(0.3) \\
0.3 \times \mathrm{SD}_{\mathrm{b}}\end{array}$ & $\%$ & $\begin{array}{c}\mathrm{MID}(0.5) \\
0.5 \times \mathrm{SD}_{\mathrm{b}}\end{array}$ & $\%$ & $\begin{array}{l}\mathrm{MID}(0.8) \\
00.8 \times \mathrm{SD}_{\mathrm{b}}\end{array}$ & $\%$ \\
\hline \multicolumn{10}{|l|}{ GMFCSI $(n=24)$} \\
\hline GMFM-88 goal total & $45.31(15.06)$ & $63.03(17.19)$ & $9.44(2.59)^{*}$ & 4.52 & 75 & 7.53 & 62.5 & 12.05 & 54.17 \\
\hline GMFM-88 total & $48.89(19.85)$ & $62.27(12.76)$ & $6.93(9.60)^{*}$ & 5.96 & 66.66 & 9.93 & 50 & 15.88 & 41.67 \\
\hline \multicolumn{10}{|l|}{ GMFCS $\|(n=15)$} \\
\hline GMFM-88 goal total & $48.58(12.54)$ & $66.47(17.76)$ & $17.89(15.58)^{*}$ & 3.76 & 66.66 & 6.27 & 60 & 10.03 & 60 \\
\hline GMFM-88 total & $50.87(17.35)$ & $63.48(12.08)$ & $12.6(12.40)^{*}$ & 5.21 & 53.33 & 8.68 & 46.67 & 13.88 & 33.33 \\
\hline \multicolumn{10}{|l|}{ GMFCS III $(n=14)$} \\
\hline GMFM-88 goal total & $36.36(17.34)$ & $48.93(18.22)$ & $12.57(10.38)^{*}$ & 5.2 & 78.57 & 8.67 & 57.14 & 13.87 & 28.57 \\
\hline GMFM-88 total & $29.46(8.77)$ & $36.26(9.08)$ & $6.80(5.20)^{*}$ & 2.63 & 92.86 & 4.39 & 78.57 & 7.02 & 21.43 \\
\hline \multicolumn{10}{|l|}{ GMFCS IV $(n=19)$} \\
\hline GMFM-88 goal total & $38.26(10.89)$ & $48.76(13.82)$ & $10.45(9.26)^{*}$ & 3.27 & 73.68 & 5.45 & 68.42 & 8.74 & 52.63 \\
\hline GMFM-88 total & $15.45(5.51)$ & $20.42(7.68)$ & $4.97(4.76)^{*}$ & 1.65 & 73.68 & 2.76 & 63.16 & 4.41 & 42.11 \\
\hline \multicolumn{10}{|l|}{ GMFCS V $(n=25)$} \\
\hline GMFM-88 goal total & $23.56(10.50)$ & $29.82(13.27)$ & $6.26(5.38)^{*}$ & 3.15 & 60 & 5.25 & 44 & 8.4 & 32 \\
\hline GMFM-88 total & $9.22(3.82)$ & $11.66(4.69)$ & $2.44(2.02)^{\star}$ & 1.15 & 64 & 1.91 & 44 & 3.06 & 32 \\
\hline
\end{tabular}

Values are presented as mean (standard deviation) and as individual MID and MID proportion indices.

GMFCS: gross motor function classification system, GMFM: gross motor function measure, MID: minimum important difference, SDb: baseline standard deviation, \%: MID proportion.

* significant differences between 1st and 2nd test, $\mathrm{p}<0.05$. 
Table 4. MID and MID proportion of GMFM-88 after intervention for CP aged 25-48 months

\begin{tabular}{|c|c|c|c|c|c|c|c|c|c|}
\hline & $\begin{array}{l}\text { 1st test } \\
\mathrm{M} \pm \mathrm{SD}\end{array}$ & $\begin{array}{l}\text { 2nd test } \\
\mathrm{M} \pm \mathrm{SD}\end{array}$ & $\begin{array}{l}\text { Change } \\
\mathrm{M} \pm \mathrm{SD}\end{array}$ & $\begin{array}{c}\mathrm{MID}(0.3) \\
0.3 \times \mathrm{SD}_{\mathrm{b}}\end{array}$ & $\%$ & $\begin{array}{l}\mathrm{MID}(0.5) \\
0.5 \times \mathrm{SD}_{\mathrm{b}}\end{array}$ & $\%$ & $\begin{array}{c}\mathrm{MID}(0.8) \\
0.8 \times \mathrm{SD}_{\mathrm{b}}\end{array}$ & $\%$ \\
\hline \multicolumn{10}{|l|}{$\operatorname{GMFCSI}(n=21)$} \\
\hline GMFM-88 goal total & $61.96(15.00)$ & $64.91(5.75)$ & $2.95(3.81)^{*}$ & 4.5 & 23.81 & 7.5 & 4.76 & 12 & 4.76 \\
\hline GMFM-88 total & $76.29(17.14)$ & 78.33 (17.73) & $2.05(2.76)^{*}$ & 5.14 & 4.76 & 8.57 & 4.76 & 13.71 & 0 \\
\hline \multicolumn{10}{|l|}{ GMFCS $\|(n=20)$} \\
\hline GMFM-88 goal total & $45.22(14.79)$ & 56.45 (19.54) & $11.23(11.69)^{*}$ & 4.44 & 68.42 & 7.4 & 42.11 & 11.83 & 35 \\
\hline GMFM-88 total & $59.51(13.49)$ & $68.26(9.15)$ & $8.75(12.07)^{*}$ & 4.05 & 73.68 & 6.75 & 36.84 & 10.79 & 20 \\
\hline \multicolumn{10}{|l|}{ GMFCS III $(n=20)$} \\
\hline GMFM-88 goal total & $43.55(12.90)$ & $52.55(15.26)$ & $9.07(10.71)^{*}$ & 3.87 & 70 & 6.45 & 40 & 10.32 & 25 \\
\hline GMFM-88 total & $49.35(9.83)$ & $55.69(9.96)$ & $6.34(6.10)^{\star}$ & 2.95 & 65 & 4.92 & 50 & 7.86 & 25 \\
\hline \multicolumn{10}{|l|}{ GMFCS IV $(n=31)$} \\
\hline GMFM-88 goal total & $40.50(12.05)$ & $45.81(12.34)$ & $5.31(4.96)^{*}$ & 3.62 & 48.39 & 6.03 & 25.81 & 9.64 & 19.35 \\
\hline GMFM-88 total & $30.21(10.79)$ & $32.96(11.10)$ & $2.74(2.72)^{*}$ & 3.24 & 25.81 & 5.4 & 12.9 & 8.63 & 6.45 \\
\hline \multicolumn{10}{|l|}{ GMFCS V $(n=22)$} \\
\hline GMFM-88 goal total & $21.41(12.19)$ & $26.25(13.02)$ & $4.84(4.94)^{*}$ & 3.66 & 40.91 & 6.1 & 27.27 & 9.75 & 13.64 \\
\hline GMFM-88 total & $8.33(5.16)$ & $10.20(5.50)$ & $1.87(1.85)^{*}$ & 1.55 & 40.91 & 2.58 & 18.18 & 4.13 & 9.09 \\
\hline
\end{tabular}

Values are presented as mean (standard deviation) and as individual MID and MID proportion indices.

GMFCS: gross motor function classification system, GMFM: gross motor function measure, MID: minimum important difference, SDb: baseline standard deviation, \%: MID proportion.

*significant differences between 1st and 2nd test, $p<0.05$.

$66.66 \%, 50 \%$, and $41.67 \%(16,12$, and 10 children) (Table 3$)$.

\section{DISCUSSION}

Maximizing functional performance and acquiring independence in the daily living environment are the main purposes of task-oriented rehabilitation therapy in children with $\mathrm{CP}^{20}{ }^{20}$ This study evaluated task-oriented training based on GMFM- 88 items in terms of clinically meaningful change after one month of training in children with CP. Children younger than 48 month of age with CP and all 5 GMFCS levels showed meaningful improvement in gross motor skill after four weeks of GMFM-based intensive task-oriented training. Specifically, MIDs of the GMFM-88-goal total score showed the most significant improvement in CP children aged $\leq 24$ months at the GMFCS levels II and III.

A pilot study by Trahan and Malouin ${ }^{21}$ for five children with CP ( 2 girls, 3 boys, mean age $=22.6$ months [SD 9.9] at GMFCS levels IV and $\mathrm{V}$ ) determined that intensive training (4 times per week for 4 weeks), followed by no training, could promote the acquisition of motor skills. The authors emphasized that a higher dose of training has to be provided over shorter periods of time in order to achieve improved motor skills in children with CP. Gannotti et al. ${ }^{22}$ reported that intervention types focused on body structures, activity, or the environment acts on a child first through the family, then through the frequency, intensity, and time to yield structural and behavioral changes. As a result, these changes are linked to improvements in functional independence.

In a 4-year longitudinal observation study of gross motor development in children with $\mathrm{CP}$, it was reported that children at all five levels continually made progress until three years of age. ${ }^{1}$ Thus, intensive and goal-directed training during the first three years of life may be crucial for meaningful changes in motor abilities.

In this study, the MIDs and the MID proportions differed according to age group and GMFCS level. The range from MID (0.3) to MID (0.8) was within the mean range of change for the respective score in children less than 48 months old. Children older than 48 months showed scores in the range between MID (0.3) and MID (0.5) for the GMFM-88-goal total score and total score. According to the results, greater improvement in motor function can be expected in children less than 48 month of age. Remarkably, children who were $\leq 24$ months of age and at GMFCS level II, seemed to be most responsive to the 1-month intensive goal-directed training. The results of this study confirm the importance of early diagnosis and early intervention in pediatric rehabilitation therapy.

More recently, MID has been used more frequently to identify a minimal level of meaningful functional change. ${ }^{23,24}$ Although MID 
could be presented as a single value, or as a range of values using a range of effect sizes (from 0.3 to 0.8 ), the latter is better than the former for clinical and research purposes. Any single value of MID may have some degree of uncertainty, while a range of MID values can provide the extent of meaningful functional change more clearly such as mild to large. ${ }^{19} \mathrm{~A}$ recent study by $\mathrm{Ko}^{12}$ assessed meaningful change after a 6-month intervention in 64 children with CP (36 boys, 28 girls, mean age $=43.8$ months) using the GMFM- 88 . Their results were presented as a range of MID values and emphasized their clinical usefulness. For example, single MID (0.3), MID (0.5), and MID (0.8) values were $4.83,8.05$, and 12.88 respectively, while the actual change score was 14.2. Given that only one of the three MID values was set for the study, we could not precisely determine how much progress had occurred during the intervention period.

In the present study, MID proportions were calculated from the MID data and used to assess each child's clinically meaningful change. Children at GMFCS levels II and III showed the highest MID proportions irrespective of their age, and the younger age group showed higher MID proportions than the older groups. Generally, as the effect sizes used to calculate MID values increased, the MID proportions decreased.

Describing a group consensus of clinical significance, Cella et al. ${ }^{25}$ reported that the use of a group MID value produced estimates that were sufficiently accurate to determine the responsiveness of individual children. Furthermore, Haley and Fragala-Pinkham ${ }^{19}$ confirmed that MID proportions were much more interpretable than a mean group change alone. In a recent study examining treatment intensity and functional mobility outcomes in 80 children with traumatic brain injury, 74\% of the participants achieved the MID value. $^{26}$

Clinicians may find advantages in using the MID proportions when documenting each child's progress, evaluating treatment effects, planning a new training program or modifying the previous program, and during discussion with caregivers. The usefulness of this practice can be demonstrated by using the results of this study. For example, in the group of children $\leq 24$ months of age at GMFCS level III $(n=14)$, the mean change score was 12.57 point and the MIDs and MID proportions were as follows: $\operatorname{MID}(0.3)=5.2$, MID (0.3) proportion $=78.57 \%$, MID $(0.5)=8.67$, MID $(0.5)$ proportion $=57.14 \%$, and $\operatorname{MID}(0.8)=13.87, \mathrm{MID}(0.8)$ proportion $=28.57 \%$. In this way, when setting the MID value as MID (0.3), 11 of the 14 chil- dren achieved a small improvement. Furthermore, eight children acquired moderate functional improvement and only four children gained a large meaningful change as a result of the intervention. According to the results of such analysis, it would be possible to rearrange our approach to training, including treatment goal, intensity, or even develop a home program.

The present study has some limitations. First, we noticed that children at levels II and III showed great improvement in all three age groups. However, the number of children at GMFCS levels II and III were relatively small and thus these results may have lower statistical power compared to Level I. A systematic follow up study, featuring larger sample sizes in each group, would be desirable to further validate a usefulness of this protocol for CP children. Second, Participant recruitment could have led to bias as there was in the present study no external randomization.

In conclusion, planning treatment together with caregivers and observing changes using a CP-specific evaluative tool, may give us important insight into the whole process of intervention including home program. For these reasons, the GMFM- 88 would be a remarkably useful strategy.

\section{REFERENCES}

1. Rosenbaum P, Paneth N, Leviton A et al. A report: the definition and classification of cerebral palsy April 2006. Dev Med Child Neurol. 2007;49(109):8-14.

2. Novak I, McIntyre S, Morgan C et al. A systematic review of interventions for children with cerebral palsy: state of the evidence. Dev Med Child Neurol. 2013;55(10):885-910.

3. Mastos M, Miller K, Eliasson AC et al. Goal-directed training linking theories of treatment to clinical practice for improved functional activities in daily life. Clin Rehabil. 2007;21(1):47-55.

4. Russell DJ, Avery LM, Rosenbaum PL et al. Improved scaling of the gross motor function measure for children with cerebral palsy: evidence of reliability and validity. Phys Ther. 2000;80(9):873-85.

5. McCarthy ML, Silberstein CE, Atkins EA et al. Comparing reliability and validity of pediatric instruments for measuring health and wellbeing of children with spastic cerebral palsy. Dev Med Child Neurol. 2002;44(7): 468-76.

6. Bania TA, Taylor NF, Baker RJ et al. Gross motor function is an important predictor of daily physical activity in young people with bilateral spastic cerebral palsy. Dev Med Child Neurol. 2014;56(12):1163-71.

7. Ketelaar M, Vermeer A, Hart H et al. Effects of a functional therapy program on motor abilities of children with cerebral palsy. Phys. Ther. 2001; 81(9):1534-45.

8. Salem Y, Godwin EM. Effects of task-oriented training on mobility function in children with cerebral palsy. NeuroRehabilitation. 2009;24(4): 
307-13.

9. Wright FV, Boschen K, Jutai J. Exploring the comparative responsiveness of a core set of outcome measures in a school based conductive education program. Child Care Health Dev. 2005;31(3):291-302.

10. Ko J, Kim M. Reliability and responsiveness of the gross motor function measure-88 in children with cerebral palsy. Phys Ther. 2013;93(3):393400 .

11. Ko JY, You YG. Reliability and responsiveness of the Korean version of the trunk impairment scale for stroke patients. J Korean Soc Phys Ther. 2015;27(4):175-82.

12. Ko J. Sensitivity to functional improvements of GMFM-88, GMFM-66, and PEDI mobility scores in young children with cerebral palsy. Percept Mot Skills. 2014;119(1):305-19.

13. Jeong DH. Reliability and validity of the CAP for computer access assessment of persons with physical disabilities. J Korean Soc Phys Ther. 2015;27(1):30-7.

14. Kim MH, Weon JH. Intra- and inter-rater reliabilities of infrasternal angle measurement. J Kor Phys Ther 2015:27(3):154-58.

15. Ko JY, Jeong JW. Reliability and concurrent validity of Korean version of the trunk control measurement scale (K-TCMS) for children with cerebral palsy. J Phys Ther Sci. 2017;29(1):16-26.

16. Seo HJ, Kim JH. The reliability and validity of Korean pediatric functional muscle testing in children with motor disorder. J Kor Phys Ther. 2016;28(4):232-42.

17. Berg KO, Norman KE. Functional assessment of balance and gait. Clin Geriatr Med. 1996;12(4):705-23.

18. Adair B, Said CM, Rodda J et al. Psychometric properties of functional mobility tools in hereditary spastic paraplegia and other childhood neurological conditions. Dev Med Child Neurol. 2012;54(7):596-605.

19. Haley SM, Fragala-Pinkham MA. Interpreting change scores of tests and measures used in physical therapy. Phys Ther. 2006;86(5):735-43.

20. Barbeau H, Fung J. The role of rehabilitation in the recovery of walking in the neurological population. Curr Opin Neurol. 2001;14(6):735-40.

21. Trahan J, Malouin F. Intermittent intensive physiotherapy in children with cerebral palsy: a pilot study. Dev Med Child Neurol. 2002;44(4): 233-39.

22. Gannotti ME, Christy JB, Heathcock JC et al. A path model for evaluating dosing parameters for children with cerebral palsy. Phys Ther. 2014; 94(3):411-21.

23. Sloan JA, Cella D, Frost MH et al. Assessing clinical significance in measuring oncology patient quality of life: introduction to the symposium, content overview, and definition of terms. Mayo Clin Proc. 2002;77(4): 371-83.

24. Angsupaisal M, Visser B, Alkema A et al. Therapist-designed adaptive riding in children with cerebral palsy: results of a feasibility study. Phys Ther. 2015;95(8):1151-62.

25. Cella D, Bullinger M, Scott C et al. Clinical significance consensus meeting group. Group vs individual approaches to understanding the clinical significance of differences or changes in quality of life. Mayo Clin Proc. 2002;77(4):384-92.

26. Dumas HM, Haley S, Carey TM et al. The relationship between functional mobility and the intensity of physical therapy intervention in children with traumatic brain injury. Pediatr Phys Ther. 2004;16(3):157-64. 